\title{
JURISPRUDENCIA AMBIENTAL INTERNACIONAL (SEGUNDO SEMESTRE 2017)
}

\author{
ROSA M. FERNÁNDEZ EGEA \\ Profesora de Derecho Internacional Público \\ Universidad Autónoma de Madrid
}


Sumario: 1. Introducción. 2. Tribunal Europeo de Derechos Humanos 3. La Corte Internacional de Justicia. 4. Organización Mundial de Comercio

\section{Introducción}

Ha transcurrido un año desde la publicación de la anterior crónica y lo cierto es que en todo este tiempo apenas contamos con pronunciamientos con implicaciones ambientales que han sido decididos o iniciados ante los principales tribunales internacionales. Los casos puramente ambientales se están librando a día de hoy, en los foros domésticos, ante los tribunales y jueces nacionales.

En el ámbito internacional, dada la inexistencia de una jurisdicción internacional consagrada a la resolución de las disputas ambientales propiamente dichas, la incidencia ambiental, a pesar de ser relevante, no suele constituir la cuestión principal de las controversias internacionales. La protección del medio ambiente ante los tribunales internacionales sigue siendo accesoria a la defensa de otros intereses, para los cuales sí existe una regulación precisa y coherente, cuyo control de cumplimiento se encuentra en manos de órganos de naturaleza judicial o arbitral. Este es el caso, por ejemplo, de la protección de los derechos fundamentales a nivel regional, tutelados por el Tribunal Europeo de Derechos Humanos o por la Corte Interamericana de Derecho Humanos; o también de los intercambios comerciales en el seno de la Organización Mundial de Comercio, que cuenta con un sistema de solución de diferencias que combina el sistema arbitral -los Grupos especiales- y de tribunal permanente -Órgano de apelación. Junto a ellos, se encuentra también la Corte internacional de Justicia que, en ocasiones, también ha de resolver controversias o emitir opiniones consultivas sobre temas que pueden tener incidencias ambientales.

En la presente crónica se hará referencia a asuntos acaecidos en dichos foros. Así, se hará referencia al avance de dos asuntos sobre los que la Corte internacional de Justicia ya se ha pronunciado, pero que seguían pendientes de conclusión, si bien por diferentes motivos: el asunto Proyecto GabcikovoNagymnaros (Hungría c. Eslovaquia) y el asunto Ciertas actividades llevadas a cabo por Nicaragua en la zona fronteriza (Costa Rica contra Nicaragua), En el ámbito de la protección regional europeo en materia de derechos fundamentales, se reseñarán tres pronunciamientos del Tribunal Europeo de Derechos 
Humanos: los asuntos Jugheli et al. c. Georgia; Otgon c. República de Moldova y S.C. Fiercolect Impex S.R.L. C. Rumania. Por último, también se informará sobre un par de asuntos presentados ante el Órgano de solución de diferencias de la Organización Mundial de Comercio que han enfrentado a dos países -India y Estados Unidos-, en una guerra comercial en relación con la implementación de ciertas medidas en el en marco de sus políticas de energía renovable: Indiadeterminadas medidas relativas a las células solares y los módulos solares y Estados Unidos - Determinadas medidas relativas al sector de la energía renovable.

Como dato curioso, cabe resaltar que de los siete casos objeto de estudio en la presente crónica, cuatro tienen alguna implicación energética: una planta térmica, una presa para generar electricidad hidráulica, y los complementos de paneles solares. Tal vez no se trate de una mera casualidad, sino que más bien, y debido a los imperativos de luchar contra el cambio climático, cada vez serán más numerosos los casos en los que los temas energéticos protagonicen las controversias internacionales con implicaciones ambientales.

\section{La Corte Internacional de Justicia}

De entre los asuntos que se anunciaron en la introducción de la presente crónica, comenzaremos por el que constituye uno de los casos más emblemáticos con implicaciones ambientales decidido por la Corte Internacional de Justicia: el asunto Proyecto Gabcikovo-Nagymnaros (Hungría c. Eslovaquia).

Este asunto trajo por causa un proyecto internacional a realizar por Hungría y la entonces Checoslovaquia, para construir una serie de presas en el rio Danubio con objeto de generar energía hidroeléctrica. En concreto se trataba de construir una presa en su paso por la ciudad eslovaca de Gabcikovo y hacer lo mismo en la ciudad húngara de Nagymaros. Con dicho fin, se celebró entre los dos países un Tratado en 1977. No obstante, pasado el tiempo, existieron opiniones enfrentadas sobre cómo implementar el Tratado, en buena forma debido al hecho de que Hungría quería terminar con el Tratado de 1977 sin haber construido su parte. Esto enfrentó a los dos países, quedando entre tanto Checoslovaquia en 1993 disuelta en dos Estados de nueva creación, la 
República checa y Eslovaquia. Esta última sería el Estado sucesor en el Tratado de 1977, sin embargo, este hecho, así como las posibles implicaciones nocivas para el medio ambiente de la construcción prevista, llevaron a Hungría a terminar unilateralmente con el Tratado de 1977. De esta forma surgió una controversia que fue sometida a la Corte Internacional de Justica mediante acuerdo de los dos países.

La Corte emitió su pronunciamiento el 25 de septiembre de $1997^{1}$, afirmando que los dos Estados debía negociar de buena fe para garantizar la consecución de los objetivos del Tratado de 1977, que seguía estando en vigor, siendo Eslovaquia el país sucesor a estos efectos.

La importancia de este asunto para el Derecho internacional del medio ambiente fue que la Corte afirmó que la protección del medio ambiente podía considerarse como un "interés esencial" en el sentido de justificar un posible estado de necesidad que evitara un ilícito internacional, y con ello, la responsabilidad internacional del Estado². Además, reafirmó el carácter consuetudinario del principio de no causar daños ambientales transfronterizos (sic uteretuo ut alienum non laedas), que ya había sido asentado en un pronunciamiento anterior (Opinión consultiva sobre la legalidad de la amenaza y uso de armas nucleares, de 8 de julio de 1996) ${ }^{3}$. No obstante, a pesar de citar alguno de los principios fundamentales del Derecho internacional del medio ambiente -principio de precaución o la obligación de evaluar los riesgos ambientales, entre otros-, lo cierto es que la Corte resolvió el asunto aplicando la máxima de "pacta sunt servanda", eso sí, indicando que las obligaciones del Tratado de 1977 tenían que ser reinterpretadas a la luz de los nuevos principios de Derecho internacional ambiental ${ }^{4}$.

\footnotetext{
1 El pronunciamiento de la ClJ puede consultarse en: http://www.icj-cii.org/files/caserelated/92/092-19970925-JUD-01-00-EN.pdf. [última consulta: 16 de octubre de 2017].

${ }^{2}$ Véase el fundamento jurídico 53, p. 41, as. Gabcikovo-Nagymnaros (Hungría c. Eslovaquia).

${ }^{3}$ La Corte afirmó: "'the environment is not an abstraction but represents the living space, the quality of life and the very health of human beings, including generations unborn. The existence of the general obligation of States to ensure that activities within their jurisdiction and control respect the environment of other States or of areas beyond national control is now part of the corpus of international law relating to the environment.", citando el fundamento jurídico 29 (pp. 241 y 242) de la Opinión consultiva sobre la legalidad de la amenaza y uso de las armas nucleares, de 1996; disponible en: http://www.icj-cij.org/files/case-related/95/095-19960708ADV-01-00-EN.pdf. [última consulta: 16 de octubre de 2017].

${ }^{4}$ Véanse los fundamentos jurídicos 111-114, pp. 64 y 65, as. Gabcikovo-Nagymnaros (Hungría c. Eslovaquia).
} 
El presente asunto, no obstante, seguía apareciendo entre las causas abiertas y pendientes en la lista de casos ante la Corte, y ello debido a que el 3 de septiembre de 1998 Eslovaquia solicitó un pronunciamiento adicional, denunciando la negativa por parte de Hungría de cumplir con la sentencia de la Corte. Por su parte, Hungría también remitió a la Corte un escrito explicando su posición. Desde entonces, las partes han estado negociando e informando puntualmente a la Corte sobre los progresos.

La construcción de la presa hidrográfica en Nagymaros nunca tuvo lugar, por lo que la presa en Gabcikovo no puede operar al máximo nivel. A la vista de que la situación no va a cambiar, Eslovaquia, en un escrito de 30 de junio de 2017 dirigido a la Corte, manifestó su deseo de poner fin a los procedimientos y de cerrar el caso definitivamente, a lo que no se ha opuesto Hungría. Así pues, la Corte informó a las partes el 18 de julio de 2017 de que ponía fin a la controversia, 24 después de que se iniciara y 20 años después de que recayera sentencia 5 .

Cabe reseñar también algún avance en un caso que sigue pendiente ante la Corte Internacional de Justicia, el asunto Ciertas actividades llevadas a cabo por Nicaragua en la zona fronteriza (Costa Rica contra Nicaragua), unido, por motivos de economía judicial, al asunto Construcción de una carretera en Costa Rica a lo largo del río San Juan (Nicaragua contra Costa Rica) ${ }^{6}$. En tales asuntos, Costa Rica y Nicaragua se reprochan mutuamente la realización de actividades con consecuencias nocivas sobre el frágil sistema ecológico del área litigiosa, en particular, sobre dos humedales de importancia internacional y protegidos por el Convenio de Ramsar?

A pesar de que la Corte Internacional de Justicia emitiera su pronunciamiento el 16 de diciembre de 2015 , este asunto sigue abierto dado a que todavía existen

\footnotetext{
${ }^{5}$ Véase la noticia sobre la terminación de la controversia en: http://www.icj-cij.org/files/caserelated/92/092-20170721-PRE-01-00-EN.pdf. [última consulta: 16 de octubre de 2017].

6 Puede verse información sobre estos asuntos http://www.icj-cij.org/en/case/150. [última consulta: 15 de abril de 2017].

7 En relación con los hechos y la sentencia de la Corte, véase la Crónica de Jurisprudencia ambiental, publicada en la RCDA, vol. 7, n.1 (2016).
} 
diferencias en relación con la metodología para establecer la compensación ordenada por la Corte, que obliga a Nicaragua a indemnizar por los daños causados por sus actividades ilícitas en el territorio costarricense. Según Nicaragua, Costa Rica pretende que le sean resarcidos unos costes desproporcionados por la ocupación nicaragüense en su territorio, entre los que incluyen algunos gastos - de policía y militares-, no previstos supuestamente en la sentencia de la Corte.

A este respecto, Costa Rica informó a la Corte el 16 de enero de 2017 de que no existía acuerdo sobre la compensación referida, fijando la Corte unos plazos para la remisión de la memoria de Costa Rica y contra memoria de Nicaragua, decidiendo mediante Providencia de 18 de julio, abrir una segunda vuelta, a la vista de las posiciones tan dispares en relación con la metodología a seguir8.

\section{Tribunal Europeo de Derechos Humanos}

Cabe recordar que, en relación con el sistema regional de protección de derechos fundamentales tutelado por el Tribunal Europeo de Derechos Humanos (TEDH), son dos los escenarios de asuntos que se presentan con incidencias ambientales ${ }^{9}$. Uno primero corresponde al grupo de casos en los que el medio ambiente se salvaguarda a través de la protección de uno o varios de los derechos fundamentales recogidos en el Convenio Europeo de Derechos Humanos (CEDH). El segundo escenario aglutina los supuestos en los que se vulnera un derecho fundamental del CEDH por razones ambientales. En la presente crónica se dará cuenta de dos asuntos que encaja en el primero de los escenarios, y un tercero que lo hace en segundo.

En relación con los casos del primer escenario, se comenzará por el de mayor importancia: el asunto Jugheli et al. c. Georgia, de 13 de julio de $2017^{10}$. En este

\footnotetext{
${ }^{8}$ El texto de la Providencia de 18 de julio puede consultarse en: http://www.icj-cij.org/files/caserelated/150/150-20170718-ORD-01-00-EN.pdf. [última consulta: 16 de octubre de 2017]. Las actuaciones llevadas a cabo en el marco de este asunto se encuentran en: http://www.icjcij.org/en/case/150. [última consulta: 16 de octubre de 2017].

${ }^{9} \mathrm{La}$ jurisprudencia del TEDH puede consultarse en: http://hudoc.echr.coe.int. [última consulta: 15 de octubre de 2017].

10 Este pronunciamiento está disponible en: https://hudoc.echr.coe.int/eng\#["fulltext":["Jugheli"],"documentcollectionid2":["GRANDCHAMBE R","CHAMBER"],"itemid":["001-175153"]\}. [última consulta: 16 de octubre de 2017].
} 
caso se trataba de una central de energía térmica que comenzó a estar operativa en 1939, construyéndose un bloque de viviendas en sus inmediaciones en el año 1952. Desde entonces, los vecinos de dichas viviendas estuvieron expuestos a una contaminación ambiental severa que conllevaba contaminación atmosférica y acústica, además de contaminación electromagnética.

La central térmica fue en un primer momento de titularidad pública y posteriormente privatizada, no obstante, nunca se sometió a una regulación especial ni se pusieron los medios de amortiguamiento de las contaminaciones, por lo que los habitantes del edificio colindante vieron mermadas su salud y calidad de vida. Es así que acudieron al TEDH alegando una vulneración del artículo 8 de la CEDH al considerar que las emisiones tóxicas no sólo constituían un agravio al medio ambiente, sino también a su salud.

El Tribunal comenzó su pronunciamiento recordando que el artículo 8 de la CEDH no puede invocarse cada vez que exista un agravio ambiental porque esta Convención no reconoce un derecho explícito a un medio ambiente sano y limpio. Sin embargo, cuando un particular se ve afectado de manera directa y seria por una contaminación acústica o de otro tipo, sí puede constituir un atentado a su derecho a disfrutar de su domicilio, su vida privada y familiar, tal y como garantiza el citado artículo 8 (fto.jdco. 62).

Citando jurisprudencia anterior, el TEDH afirmó que, para que exista una vulneración del artículo 8 no cabe probar cualquier injerencia, sino que es necesario superar una regla de minimis. El listón de lo que supondría una interferencia "razonable" deberá establecerse caso por caso, atendiendo a las circunstancias del supuesto concreto, en particular, teniendo en cuenta la intensidad y la duración de la contaminación, así como la severidad de los daños (físicos y psicológicos) causados a los particulares. Ello con objeto de discernir si tales inconvenientes son los propios de la vida moderna en las ciudades, que pueden ser tolerados, de otras injerencias que pueden afectar gravemente al bien-estar de los individuos y disfrute de sus vidas privadas y domicilios (fto.jdco. $62)$.

Además, también recordó que el Estado no sólo está obligado a refrenarse de llevar a cabo tales interferencias perjudiciales para la calidad de vida de sus 
administrados, sino que también cuenta con una obligación positiva de adoptar las medidas necesarias para que terceras personas no produzcan tales interferencias en menoscabo de otros (fto.jdco. 64).

Si bien es cierto que el Estado cuenta con un margen de apreciación para regular las actividades que pueden atentar contra la vida privada y familiar, el TEDH puede entrar a valorar si existe un equilibrio equitativo entre los intereses que se encuentran enfrentados, en este caso, la generación de energía eléctrica de la que se beneficia la ciudad, por un lado, y la salud y vida privada de los vecinos, por otro. En este sentido, el Tribunal constató los graves problemas de salud de los reclamantes y el hecho de que éstos no eran conscientes cuando entraron a vivir al edificio de los riesgos que podía entrañar el funcionamiento de la planta vecina. Pero, lo determinante para considerar que el Estado falló en establecer un balance justo entre los intereses en juego fue que no se había adoptado ningún tipo de legislación (en materia de licencias, seguridad, supervisión, evaluación de riesgos, etc.) que sería requerida para una actividad con tales repercusiones para el medio ambiente y la salud de las personas, ni se había exigido la instalación de sistema alguno de contención de la contaminación atmosférica y acústica. De esta forma, la actitud pasiva adoptada por el Estado ante una actividad tan dañina, sin adoptar medidas de prevención de riesgos, ha constituido una nueva vulneración del artículo 8 de la CEDH, que viene a engrosar una ya muy nutrida jurisprudencia al respecto.

El segundo de los asuntos que pueden subsumirse en el primer escenario es de menor importancia por cuanto la vulneración de la vida privada ya había sido constada por el Estado infractor en sede nacional. No obstante, es interesante reseñarlo para evidenciar el concepto tan amplio que maneja el TEDH de "vida privada" y que ha permitido poder conocer de una multitud de casos con implicaciones ambientales, dando protagonismo al medio ambiente, aunque sea a través de la defensa de un derecho fundamental individual. 
Se trata del asunto Otgon c. República de Moldova, de 25 de octubre de $2016^{11}$, que surgió con motivo de una intoxicación que sufrieron una mujer y su hija tras beber agua del grifo contaminada. Efectivamente, quedó probado que debido a unas filtraciones de una tubería de agua de alcantarillado en la tubería del agua potable, las reclamantes sufrieron serios problemas de salud, permaneciendo varias semanas en el hospital. La Administración responsable se hizo cargo de la responsabilidad de tales hechos, indemnizando a las víctimas, aunque, a juicio de estas últimas, de manera insuficiente. Tras agotar la vía nacional, acudieron al TEDH, reclamando una mayor indemnización, en aplicación del artículo 8 del CEDH.

El Tribunal volvió en este asunto a recordar su jurisprudencia en relación con el artículo 8 del CEDH, resaltando que no existe un derecho individual a un medio ambiente sano, pero que cuando la interferencia con la vida privada y familiar es de tal magnitud, puede constituir una vulneración de este precepto (fto.jdco. 15). Esto puede afirmarse también cuando un Estado no cumple con su obligación positiva de adoptar todas las medidas necesarias para evitar tal interferencia por un medio ambiente poco saludable, como fue el caso (hecho no discutido por el Estado demandado).

La peculiaridad de este caso es que la parte demandante considera que se vulnera el artículo 8, no por los hechos anteriormente narrados, sino porque la indemnización por los mismos fue insuficiente (una cantidad equivalente a 310 euros). Argumento que convenció al Tribunal, tras constatar que la indemnización obtenida era muy inferior a la cantidad que, en circunstancias similares, suele decidir el propio Tribunal en aplicación del artículo 41 de la $\mathrm{CEDH}$. Ahora bien, frente a los 50.000 euros que solicitaba la parte reclamante, el Tribunal sólo le concedió 4.000 , aun así, una cantidad muy superior a la obtenida en sede nacional.

Este pronunciamiento, atípico por centrarse exclusivamente en la consideración de la compensación, ha motivado una Opinión disidente del Juez Lemmens. El

\footnotetext{
11 Este pronunciamiento está disponible en: https://hudoc.echr.coe.int/eng\#\{"fulltext":["Otgon"],"documentcollectionid2":["GRANDCHAMBER ","CHAMBER"],"itemid":["001-167797"]\}. [última consulta: 15 de octubre de 2017]
} 
magistrado del TEDH considera que se trata de un caso en el que se discute el derecho a ser compensado por daños sufridos, independientemente de lo que motivó dichos daños. En su escrito, el juez disidente concluye que no está de acuerdo con una ampliación tan "generosa" de la noción de "vida privada", que permite elevar de estatus un simple caso de responsabilidad civil (extracontractual del Estado, se entiende) a uno de relevancia "constitucional", por afectar a derechos fundamentales. Sin embargo, ¿no afecta también a los derechos fundamentales de las reclamantes el hecho de haber sido insuficientemente indemnizadas a la vista de los graves perjuicios que se causó a su salud?

Respecto al segundo de los escenarios, se trata, como suele ser habitual, de considerar si existe vulneración del derecho de propiedad recogido en el artículo 1 del Protocolo 1 al CEDH, cuando éste ha sido objeto de injerencias por razones ambientales.

Se trata del asunto S.C. Fiercolect Impex S.R.L. C. Rumania, de 13 de diciembre de $2016^{12}$, que concierne a una empresa que se dedica a recolectar y reciclar desechos de acero, como principal actividad. La empresa estuvo realizando dicha actividad amparada con un permiso que caducó en marzo de 2005. Tras solicitar su prórroga, fue informada de que tenía que solicitar un nuevo permiso conforme a la legislación ambiental recientemente adoptada, dado el impacto importante para el medio ambiente de la actividad que realizaba. Así lo hizo la empresa, obteniendo el nuevo permiso en abril de 2005. El problema es que entre la fecha de caducidad del anterior permiso (7 de marzo) y la obtención del nuevo permiso (14 de abril), siguió con la actividad, tal y como se constató en una inspección realizada en la sede de la empresa un mes después.

A la vista de esta actuación ilegal, la empresa tuvo que soportar una multa por un monto equivalente a 694 euros, a la vez que se le confiscó una cantidad equivalente a algo más de 21.000 euros, que representaba el valor de los desechos de acero obtenidos durante el periodo en el que operó sin permiso. La 
empresa inició los procedimientos contra dichas medidas que, a su juicio, atentaban contra su derecho de propiedad (sobre todo en relación con la medida confiscatoria) y que se debían, a su juicio, a un retraso indebido de la emisión del permiso correspondiente por la Administración de su país. Tras agotar la vía nacional, llegó el asunto ante el TEDH, invocando principalmente la vulneración del artículo 1 del Protocolo 1 a la $\mathrm{CEDH}$, que consagra el derecho de propiedad privada.

En su pronunciamiento, el TEDH recordó, lo que ya es jurisprudencia asentada, que el artículo 1 del Protocolo 1 a la CEDH consta de tres reglas diferentes que hay que verificar para constatar su vulneración (fto.jdco. 55) ${ }^{13}$ :

— La primera regla se encuentra contenida en la primera frase del primer párrafo, que enuncia el principio del disfrute pacífico de la propiedad.

- La segunda regla, recogida en la segunda frase del primer párrafo, se consagra a la privación de la posesión, sometiéndola a distintas condiciones.

- La tercera regla, fijada en el segundo párrafo, reconoce que los Estados pueden introducir injerencias en el derecho de propiedad si lo hacen de acuerdo a un interés general.

Como suele ser habitual, en el presente caso no se cuestionaba la existencia de una injerencia sobre el derecho al disfrute pacífico de sus posesiones (derecho de propiedad), ni que la medida confiscatoria encajase en el supuesto previsto en el segundo párrafo del artículo 1, anteriormente referido. Lo que el Tribunal tenía que comprobar es si dicha injerencia estaba justificada y para ello debía determinarse si se cumplía los requisitos asentados previstos en el segundo párrafo y que en su jurisprudencia ha concretado a los siguientes: si la injerencia

\footnotetext{
${ }^{13}$ El artículo 1 del Protocolo 1 al CEDH establece lo siguiente:

Protección de la propiedad.

Toda persona física o moral tiene derecho al respeto de sus bienes. Nadie podrá ser privado de su propiedad más que por causa de utilidad pública y en las condiciones previstas por la Ley y los principios generales del derecho internacional.

Las disposiciones precedentes se entienden sin perjuicio del derecho que poseen los Estados de poner en vigor las Leyes que juzguen necesarias para la reglamentación del uso de los bienes de acuerdo con el interés general o para garantizar el pago de los impuestos $u$ otras contribuciones o de las multas.
} 
tiene base legal, si se realiza atendiendo a un interés general y si existe proporcionalidad (fto.jdco. 57 in fine).

El primer requisito, que la interferencia tenga base legal, no es más que una manifestación del Estado de Derecho (rule of law), que, como afirma el Tribunal, es un principio fundamental en una sociedad democrática (fto.jdco. 58). En el presente caso, no existían discrepancias sobre este hecho pues la multa y la confiscación del dinero fueron impuestas tras una aplicación fehaciente de lo establecido en la legislación vigente. En cuanto a si dicha legislación obedecía a la protección de un interés general, éste tampoco parecía discutirse, pues se trataba de asegurar el control estatal sobre las condiciones que determinadas actividades empresariales han de cumplir dado su gran impacto sobre el medio ambiente (fto.jdco. 60).

Dicho esto, lo que restaba examinar al TEDH era, como suele ser también habitual en los casos de este tipo que llegan a su conocimiento, comprobar si existía un equilibrio justo entre las demandas del interés general, y el derecho de propiedad del particular. En otras palabras, si la imposición de la multa y medida confiscatoria eran medidas proporcionadas con los fines que pretendían seguir e intereses que pretendían proteger (ftos.jdcos. 61 y ss.). A este respecto, el TEDH se refirió a que la importancia de proteger el medio ambiente se ha visto acrecentada en las últimas décadas y es una cuestión que no puede ceder ante otro tipo de imperativos, como los de naturaleza económica o financiera (fto.jdco. 65). Las autoridades públicas no sólo tienen que garantizar esta protección, sino que también han de velar por que ésta sea eficaz, lo que no se cumpliría tratándose de una multa con un monto irrisorio. El Tribunal recordó que en estos casos, el Estado tiene un amplio margen de apreciación, incluso para decidir que las conductas ilegales que puedan entrañar perjuicios ambientales se castiguen con una multa en conjunción con una medida confiscatoria (ftos.jdcos. 67 y 68). En opinión del TEDH, tales medidas no eran desproporcionadas porque sólo se confiscó un monto de dinero equivalente a los beneficios obtenidos por la empresa por la realización de actividad durante el periodo no cubierto por el permiso administrativo. Esto, a su juicio, no supuso una carga excesiva para el particular y, por consiguiente, no hubo vulneración del artículo 1 del Protocolo 1 del CEDH (ftos.jdcos. 71-73). 


\section{Organización Mundial de Comercio}

En relación con las diferencias acaecidas en el marco de la Organización Mundial del Comercio, en ocasiones se encuentran controversias comerciales que tienen implicaciones ambientales, aunque es raro el asunto en el que la preocupación ambiental puede motivar una restricción al comercio, hasta el punto de justificar la vulneración de una obligación comercial ${ }^{14}$. Durante el periodo de tiempo que comprende la presente crónica contamos con dos asuntos, uno ya decido - el asunto India-determinadas medidas relativas a las células solares y los módulos solares- y otro que acaba de iniciarse -el asunto EE.UU.-determinadas medidas relativas al sector de la energía renovable-. Los dos afectan a dos países, India y EE.UU. (aunque también se han unido a la causa otros países en calidad de terceros), que se intercambian los papeles de Estado reclamante y Estado reclamado en lo que parece ser una "guerra comercial" en relación con la forma que tienen de implementar sus políticas de incentivación de la energía renovable. En primer lugar, el asunto India-determinadas medidas relativas a las células solares y los módulos solares (DS456) ${ }^{15}$, se inició con la solicitud de EE.UU. de celebrar consultas con la India en relación con las medidas que esta última había adoptado en el marco de su programa de energía solar (llamado National Solar Mission - en adelante, $\mathrm{NSM})^{16}$. En el marco del NSM, para incentivar la producción de energía solar, el gobierno hindú se comprometía a comprar la energía generada de las plantas de energía solar, y distribuirla a los consumidores. Sin embargo, sólo podían participar en el NSM aquellos productores que utilizaran células y módulos solares de fabricación nacional. A juicio de EE.UU., el NSM establecía un trato discriminatorio respecto de los módulos solares importados, a favor de los nacionales, lo que contravenía, entre

\footnotetext{
${ }^{14}$ Hasta la fecha sólo ha ocurrido en una ocasión y fue con objeto de una medida cuyo objeto era salvaguardar la salud de las personas (el asunto CE.-Medidas que afectan al amianto y a productos que contienen amianto -DS135-, de 2000).

${ }^{15}$ La información relevante sobre esta controversia está disponible en la página Web de la OMC: https://www.wto.org/english/tratop e/dispu e/cases e/ds456 e.htm. [última consulta: 15 de octubre de 2017]

${ }^{16}$ La celebración de consultas es el primer paso en la resolución de disputas en el seno de la OMC. Véase el Entendimiento de Solución de Diferencias, disponible en: https://www.wto.org/spanish/docs s/legal s/28-dsu s.htm. [última consulta: 15 de octubre de 2017]
} 
otras disposiciones ${ }^{17}$, el artículo III.4 del Acuerdo General sobre Aranceles Aduaneros (GATT, por sus siglas en inglés).

Como las consultas resultaron infructuosas, el 23 de mayo de 2014 se estableció un Grupo especial que conocería del caso para darle una solución vinculante para las partes. El informe del Grupo especial se emitió el 24 de febrero de 2016, confirmado las alegaciones de EE.UU., al considerar que la India había vulnerado sus obligaciones comerciales por cuanto las medidas adoptadas en el seno del NSM constituían un trato menos favorable de productos importados respecto de "productos similares" nacionales, contrario al artículo III.4 GATT.

No se podía aplicar en este caso, como pretendía la India, la excepción prevista en el apartado 8 a) del mismo artículo III GATT, que da cobertura a las medidas que "rijan las adquisición, por organismos gubernamentales, de productos comprados para cubrir las necesidades de los poderes públicos y no para su reventa comercial". A este respecto, el Grupo Especial, citando un caso precedente (Canadá-Energía renovable), advirtió que los "productos comprados" a los que hacía referencia la excepción era la energía solar, y no las células y paneles solares, que son los productos sobre los cuales se establecía la discriminación prohibida por el artículo III.4 GATT.

La India invocó igualmente la aplicación de dos excepciones previstas en el artículo XX del GATT, concretamente las contenidas en los apartados d) y j), pero sin éxito. Este artículo contiene una serie de excepciones que, atendiendo a objetivos de interés público, admiten las restricciones al comercio y justifican así una posible vulneración de la normativa GATT.

El apartado d) del artículo XX está previsto para justificar las medidas "necesarias para lograr la observancia de las leyes y de los reglamentos que no sean incompatibles con las disposiciones" del GATT. La India invocó esta excepción argumentando que tales medidas se adoptaban para promover el desarrollo sostenible y sus compromisos internacionales en el marco del reciente Acuerdo de París sobre cambio climático. A este respecto, si bien el Grupo

\footnotetext{
${ }^{17}$ Otras de las disposiciones que se vulneraban, según los EE.UU., eran el art.2.1 del Acuerdo sobre medidas en materia de inversiones relacionadas con el comercio - Acuerdo MIC-, y otros artículos -3, 5, 6 y 25- del Acuerdo sobre subvenciones y medidas compensatorias - Acuerdo SMC-. Sin embargo, la controversia se resuelva básicamente con las obligaciones contenidas en el GATT.
} 
Especial afirmó que los acuerdos internacionales pueden constituir las "leyes" y "reglamentos" a los que hace mención esta disposición, es necesario que exista un efecto directo en el ordenamiento jurídico nacional del país. Es decir, es preciso determinar el carácter normativo y vinculante de tales regulaciones ambientales a nivel nacional, así como que sean directamente invocables ante sus tribunales y administraciones públicas. Estas características son difíciles de cumplir habida cuenta la vaguedad y poca concreción con la que están definidas las obligaciones en el Acuerdo de París y la Convención Marco de Naciones Unidas sobre Cambio Climático. Por otra parte, esta decisión no sorprende por cuanto los órganos de solución de diferencias de la OMC suelen ser reacios a aplicar normas de Derecho internacional ambiental para resolver las controversias ambientales ${ }^{18}$.

Tampoco parecía cumplirse el supuesto previsto en el párrafo j) del artículo XX que justifica las medidas "esenciales para la adquisición o reparto de productos de los que haya una penuria general o local". India sostuvo que las medidas controvertidas estaban justificadas al amparo de esta excepción porque su falta de capacidad nacional de fabricación de células y módulos solares las cualificaba como productos sobre los que cabía afirmar que existía una "penuria general o local". Sin embargo, a juicio del Grupo especial, esto sería el caso de una situación en la que la cantidad de abastecimiento de un producto no puede satisfacer las demandas de la población, sin que la India hubiera podido probar que tan siquiera existía un riesgo de que esto pudiera ser así. Es más, también tendrían que tenerse en cuenta la posibilidad de abastecimiento internacional, que era precisamente lo que India intentaba evitar con el programa NSM.

Es curioso que, tratándose de justificaciones con ciertas pretensiones ambientales, la India no hubiera invocado las dos excepciones recogidas en el artículo XX GATT que tradicionalmente han dado cobertura a restricciones sobre el comercio por motivos ambientales: los apartados b) y g), que contemplan las

\footnotetext{
${ }^{18} \mathrm{~A}$ modo de ejemplo, ni el principio de precaución ni el Protocolo de Cartagena sobre organismo vivos modificados pudieron ser relevantes para la resolución del asunto CE-Comunidades Europeas - Medidas que afectan a la aprobación y comercialización de productos biotecnológicos (DS261), en el sentido que evidenciaban que los productos genéticamente manipulados no eran "similares" a los productos convencionales, deshaciendo de esta forma, la discriminación alegada.
} 
medidas "necesarias para proteger la salud y la vida de las personas y de los animales o para preservar los vegetales" y "relativas a la conservación de los recursos naturales agotables", respectivamente. Esto pudiera llevar a pensar que, en realidad, las motivaciones que subyacen en su normativa eran más de naturaleza proteccionista de la producción nacional de células y paneles solares, que verdaderamente ambientales.

No obstante, el programa NSM también podría verse como un incentivo no sólo a la generación de energía solar en la India, que le permita cumplir con sus compromisos internacionales en materia de cambio climático, sino también a la producción de células y módulos solares de origen nacional, con miras a crear un mercado que permita abastecer sus plantas solares sin necesidad de depender de las importaciones de terceros Estados. No en vano, el autoabastecimiento energético, sobre todo cuando éste es de naturaleza renovable, bien parece un objetivo loable y digno de protección frente a otros intereses de carácter comercial. Máxime cuando se trata de un país como la India con una población ingente y creciendo a velocidad exponencial, constatándose que muchas de sus comunidades carecen a día de hoy de acceso a la electricidad.

La India apeló el informe del Grupo especial, pero el Órgano de Apelación confirmó, en su pronunciamiento de 16 de septiembre de 2016, las constataciones realizadas por el primero.

En lo que parece ser una respuesta a la controversia que enfrentó a la India y a EE.UU., ha llevado al primero a iniciar una controversia ante la OMC frente al segundo, también por medidas supuestamente discriminatorias adoptadas en algunos de sus Estados federados en el sector de la energía: EE.UU.determinadas medidas relativas al sector de la energía renovable (DS 510) ${ }^{19}$.

El 9 de septiembre de 2016, la India solicito la celebración de consultas con los EE.UU. en relación a determinas medidas relativas a prescripciones de contenido nacional y subvenciones de los gobiernos de los Estados de Washington, California, Montana, Massachusetts, Connecticut, Michigan,

\footnotetext{
${ }^{19}$ La información relevante sobre esta controversia está disponible en la página Web de la OMC: https://www.wto.org/english/tratop e/dispu e/cases e/ds510 e.htm. [última consulta: 15 de octubre de 2017]
} 
Delaware y Minnesota; argumentando la vulneración de prácticamente las mismas disposiciones comerciales que las invocadas en el asunto anteriormente mencionado. En la fecha en la que se concluye esta crónica, todavía no se ha decidido la constitución de un Grupo especial que decida el caso, no obstante, estaremos pendientes para informar en un futuro sobre este particular. De constatarse la discriminación denunciada por la India, sería paradójico que EE.UU. haya cuestionado el programa de energía solar hindú, llevándose a cabo políticas igualmente proteccionistas de fomento de energías renovables en su territorio. 